\title{
Single Beat RR Extrapolated Heart Rate by ECG Assessment
}

National Cancer Institute

\section{Source}

National Cancer Institute. Single Beat RR Extrapolated Heart Rate by ECG Assessment. NCl Thesaurus. Code C120608.

The extrapolation of an individual's heart rate as estimated from a single R-R interval as measured by an electrocardiog raphic machine. 